\title{
Resiliencia en estudiantes universitarios de educación de Huancayo
}

\section{Resilience in university students of education from Huancayo}

\author{
aBaltazar Castañeda, L.; Lazo Piñas, M.; \\ Casallo Poma, L.; Gómez Galindo, M. \\ Facultad de Educación / Universidad Nacional del Centro del Perú \\ Email: Ibaltazar@uncp.edu.pe
}

\section{Resumen}

El presente estudio, aborda el tema de la resiliencia humana, considerando al enfoque ecológico de desarrollo humano. Este enfoque centra su esfuerzo en analizar el comportamiento, atendiendo a su multicausalidad, en la que factores externos e internos se interconjugan haciendo del ser humano un sujeto, cuyas cualidades y capacidades internas, se ven fuertemente influencias por el entorno o contexto. La investigación internaliza las características de un estudio de corte cuantitativo realizado con el método comparativo con acotaciones descriptivas.

Se evaluaron, de forma no probabilística, a 448 estudiantes de las facultades de educación de dos universidades de la ciudad de Huancayo: UNCP y UPLA. Para la medición de la resiliencia, se adaptó la escala de ER de Wagnild y Young. Los resultados indican mayor predominio de estudiantes con nivel medio de resiliencia. A nivel comparativo, se logró precisar que el tipo de gestión de la universidad si es un factor diferencial influyente en la configuración del perfil de resiliencia de los estudiantes investigados, lo que permitió observar mayores niveles de resiliencia en los estudiantes procedentes de universidad privada. En cambio, el género no se muestra como un factor potencialmente diferenciador sobre la resiliencia de los jóvenes estudiados.

Palabras clave: resiliencia, estudiante universitario, enfoque ecológico, universidad, psicología positiva

\begin{abstract}
The study presented addresses the issue of human resilience considering the ecological approach to human development. This approach focuses its effort on analyzing the behavior according to its multi-causality, in which external and internal factors interconjugan making the human being a subject, whose inner qualities and capabilities are strongly influenced by the environment or context. The study internalizes the characteristics of a quantitative study carried out with the comparative method with descriptive dimensions.

A total of 448 students from the faculties of education of two universities in the city of Huancayo were evaluated (nonprobabilistically): UNCP and UPLA. For the measurement of Resilience, the Wagnild and Young ER Scale was adapted. The results indicate a greater predominance of students with an average level of resilience. At a comparative level it was possible to specify that the type of management of the university is an influential differential factor in the configuration of the resilience profile of the students investigated, which allowed to observe higher levels of resilience in the students coming from private universities. In contrast, gender is not shown as a differential factor in the evaluated psychosocial variable.
\end{abstract}

Keywords: resilience, university student, ecological approach, university, positive psychology 


\section{Introducción}

El término de la resiliencia es un constructo que deviene del enfoque salugénico, como un abordaje reciente de la psicología positiva. Hace referencia a los factores que evocan al comportamiento de las personas que se sobreponen de manera positiva y saludable frente a las situaciones o dificultades que para otras serían frustrantes (Arratia, Medina y Borja, 2008).

No obstante, las diferencias al respecto, se ha llegado a un acuerdo en lo referente al entendimiento del término de resiliencia, pues la mayoría de los estudiosos (Arratia, Medina y Borja, 2008; Pereira, 2007 y Polo, 2009) la consideran como una capacidad humana para lograr el éxito o sobrellevar de un modo favorable y adaptativo las situaciones frustrantes o estresantes que podrían tener consecuencias negativas en el individuo.

Así, Pereira (2007) afirma que la resiliencia es un proceso dinámico. En la psicología es considerada como una capacidad de los individuos de sobreponerse a condiciones de vida desfavorables para tener éxito (Puerta y Vásquez, 2012).

La resiliencia puede ser analizada desde dos enfoques distintos. Por un lado, considerando la perspectiva de "riesgo", la cual se concentra en los aspectos que pueden desencadenar un daño psicológico, biológico o social, su interés se encuentra en los factores de riesgo alrededor del individuo. Por otro lado, está el enfoque de resiliencia o "modelo de desafío", propone la existencia de aspectos o escudos protectores que pueden generar una defensa al posible daño o amenaza, de los ya mencionados factores de riesgo, y que provocan en el individuo la capacidad o la habilidad para superar las adversidades (Polo, 2009).

La integración de estos dos enfoques, permiten tener una visión más clara y complementaria del fenómeno. En tal sentido, se propone, por un lado, la identificación de las variables que pueden afectar negativa y considerablemente al individuo; así mismo, se contempla la existencia de barreras que pueden contribuir a sobrellevar situaciones riesgosas e inclusive, a sacarles el mayor provecho a la adversidad, considerando aspectos positivos, lo cual tiene por consecuencia que el individuo desarrolle y mejore su calidad de vida.

Las investigaciones sobre resiliencia han sido desarrolladas en diversos contextos (Börkan, Erkman y Serbest, 2016; Schofield, O’Halloran, Mclean y Forrester-Knauss, 2016; y, Wang, Chen, Gong y Yan, 2016), poblaciones, ámbitos de estudio e incluso en distintas etapas de la vida humana.

Estudios recientes dentro del grupo de adolescentes, como las de Álvarez, Alonso y Guidorizzi (2014); Gutiérrez y Romero (2014); Morales y González (2014); Rozemberg, Avanci, Schenker y Pires (2014); Quiceno, Mateus, Villareal y Vinaccia (2013); y, González y Gaxiola (2013) vinculan a la resiliencia con el consumo de drogas, bienestar psicoló- gico, autoestima, y variables sociodemográficas como factores que afectan esta capacidad dentro del ámbito escolar.

Dentro de estas últimas acotaciones aparece el término de resiliencia académica. Centrando estas líneas en la población joven dentro del ámbito escolar, se presenta un abordaje de las investigaciones en este contexto, específicamente investigaciones vinculadas con estudiantes de carreras universitarias.

Los estudios muestran mayormente interés en la relación de la resiliencia, con variables tales como; condiciones de estrés, esfuerzo y exigencia propios de los estudios superiores. Ríos, Carrillo y Sabuco (2012), por ejemplo, investigaron la relación de la resiliencia con el síndrome Burnout en una muestra de alumnos de enfermería en España, encontrando que solo un pequeño porcentaje de la población de la muestra $(14,7 \%)$ obtuvo bajos niveles de resiliencia. Del mismo modo, se identificaron los puntajes más bajos de Burnout en los estudiantes que denotaron altos indicadores de resiliencia, así como menores signos de cansancio emocional y mayor realización personal.

González y Artuch (2014) también encontraron altos niveles de resiliencia en estudiantes universitarios, así como una asociación con el sexo y el tipo de universidad, siendo las mujeres de instituciones de educación religiosa, quienes obtuvieron puntajes mayores. Por otro lado, Schofield, O'Halloran, Mclean y Forrester-Knauss (2016) identificaron la resiliencia como un factor inversamente proporcional a la conducta depresiva en estudiantes universitarios en Australia. Tenemos a Rivas (2012) quien analizó el perfil de las fortalezas de carácter y los factores de resiliencia de los estudiantes de medicina en Venezuela, para lo cual consideró, como factores de resiliencia, a la aceptación positiva al cambio, la competencia personal, el control y la espiritualidad.

Estudio del cual obtuvo como resultados que los estudiantes de medicina de Venezuela poseen los cuatro factores de la resiliencia. De acuerdo a las variables personales, se identificó que la capacidad resiliente de los estudiantes más jóvenes era mayor a las de jóvenes de mayor edad; además, de presentarse una relación inversa con el grado académico y con la presencia de hijos en años escolares. A esto, en su investigación, Álvarez y Cáceres (2010), sobre la relación de la resiliencia y las variables personales y sociodemográficas, encontraron que la correlación entre variables oscilaba entre débil y muy débil; sin embargo, se encontró diferencias significativas en cuanto al sexo de los participantes, Álvarez y Cáceres afirman que las mujeres obtuvieron mayores puntajes de resiliencia en comparación a los varones, teniendo también como resultado que los escolares solteros mostraban más indicadores de resiliencia que los escolares casados.

Velasco, Suárez, Córdova, Luna y Mireles (2015), en su investigación en México, con alumnos de enfermería tuvieron como objetivo conocer el nivel de resiliencia, 
para lo cual utilizaron la Escala de Resiliencia Mexicana (RESI-M) (Palomar \& Gómez, 2010), la cual, les permitió analizarlas haciendo una relación con otras variables personales, académicas, de salud, laborales, económicas y familiares. Tuvieron como resultado que el $63 \%$ de los estudiantes de enfermería presentaban un nivel alto de resiliencia, creado relación entre este resultado y su desempeño académico y la estructura familiar de cada participante, por que consideran a estas variables como factores dependientes para desarrollar la capacidad de resiliencia.

Gonzáles (2011) evaluó los factores resilientes de estudiantes de primer grado de medicina, en la ciudad de México, para lo cual, utilizó el Cuestionario de Resiliencia para Estudiantes Universitarios (CRE-U), que le proporcionó como resultado que, los estudiantes de medicina obtuvieron puntajes altos en las dimensiones de interacción, introspección y moralidad, así como niveles bajos en los factores de independencia, creatividad, humor y pensamiento crítico. Teniendo como aporte importante que las mujeres obtuvieron puntajes más altos que los varones.

\section{Métodos y materiales}

El presente estudio adopta una metodología con enfoque cuantitativo. Corresponde a un estudio empírico, de campo, de nivel correlacional. Como método general, se empleó al método científico; como método específico, se empleó al método comparativo. El diseño que responde a las características del estudio es el No experimental de tipo descriptivo - comparativo retrospectivo.

La muestra, consideró a 448 estudiantes de las facultades de educación de las universidades públicas y privadas de la ciudad de Huancayo del departamento de Junín. Según tipo de universidad, la muestra (no probabilística) estuvo conformada por 308 estudiantes procedentes de universidad pública y 140 estudiantes procedentes de universidad privada. Según género, 254 son varones y 194 mujeres. Para el acopio de datos de la resiliencia se empleó la "Escala de Resiliencia" de Wagnild y Young; adaptada, validada y confiabilizada para el caso.

Tabla 1

$V$ de Aiken de la Escala de Resiliencia - Adaptada

\begin{tabular}{lcccccc}
\hline \multirow{2}{*}{ Indicadores } & \multicolumn{5}{c}{ Jucio valorativo (Jueces) } & Coeficiente \\
\cline { 2 - 6 } & $\mathbf{1}$ & $\mathbf{2}$ & $\mathbf{3}$ & $\mathbf{4}$ & $\mathbf{5}$ & de Aiken* \\
\hline Relevancia & $\mathrm{Si}$ & $\mathrm{Si}$ & $\mathrm{Si}$ & $\mathrm{Si}$ & $\mathrm{Si}$ & 1,00 \\
Adecuación & $\mathrm{Si}$ & $\mathrm{Si}$ & $\mathrm{Si}$ & $\mathrm{Si}$ & $\mathrm{Si}$ & 1,00 \\
Suficiencia & $\mathrm{Si}$ & $\mathrm{Si}$ & $\mathrm{Si}$ & $\mathrm{Si}$ & $\mathrm{Si}$ & 1,00 \\
Objetividad & $\mathrm{Si}$ & $\mathrm{Si}$ & $\mathrm{Si}$ & $\mathrm{Si}$ & $\mathrm{Si}$ & 1,00 \\
Funcionalidad & $\mathrm{Si}$ & $\mathrm{Si}$ & $\mathrm{Si}$ & $\mathrm{Si}$ & $\mathrm{No}$ & 0,80 \\
Pertinencia & $\mathrm{Si}$ & $\mathrm{Si}$ & $\mathrm{Si}$ & $\mathrm{Si}$ & $\mathrm{Si}$ & 1,00 \\
Mensuración & $\mathrm{Si}$ & $\mathrm{Si}$ & $\mathrm{Si}$ & $\mathrm{Si}$ & $\mathrm{Si}$ & 1,00 \\
\hline
\end{tabular}

Conforme a la opinión de los jueces, el instrumento empleado cuenta con la validez de contenido acceptable.

Tabla 2

Validez discriminante de la Escala de Resiliencia - Adaptada

\begin{tabular}{cccc}
\hline Items & $\begin{array}{c}\text { Correlación } \\
\text { elemento-total } \\
\text { corregida }\end{array}$ & Items & $\begin{array}{c}\text { Correlación } \\
\text { elemento-total } \\
\text { corregida }\end{array}$ \\
\hline VAR1 & 0,349 & VAR14 & 0,404 \\
VAR2 & 0,348 & VAR15 & 0,475 \\
VAR3 & 0,567 & VAR16 & 0,496 \\
VAR4 & 0,371 & VAR17 & 0,539 \\
VAR5 & 0,484 & VAR18 & 0,414 \\
VAR6 & 0,285 & VAR19 & 0,442 \\
VAR7 & 0,318 & VAR20 & 0,383 \\
VAR8 & 0,430 & VAR21 & 0,455 \\
VAR9 & 0,490 & VAR22 & 0,293 \\
VAR10 & 0,494 & VAR23 & 0,432 \\
VAR11 & 0,455 & VAR24 & 0,383 \\
VAR12 & 0,435 & VAR25 & 0,513 \\
VAR13 & 0,520 & & \\
\hline
\end{tabular}

Se determinó el valor del estadístico de fiabilidad para 25 elementos, alfa de Cronbach igual a 0,873.

\section{Resultados}

\section{Diagnóstico del resiliencia en la muestra total}

En el conglomerado, del $100 \%(n i=498)$ de estudiantes universitarios de educación encuestados: el $18 \%$ (81) se autopercibe con resiliencia de nivel alto, el $59 \%$ (254) obtuvo puntuaciones que reflejan una resiliencia de nivel medio y el $23 \%$ (103) obtuvieron calificaciones que describen la presencia de un nivel bajo de resiliencia, considerada como una fortaleza personal.

\section{Tabla 3}

Niveles de resiliencia en la muestra total

\begin{tabular}{lcc}
\hline \multirow{2}{*}{ Resiliencia } & \multicolumn{2}{c}{ Muestra total global } \\
\cline { 2 - 3 } & Ni & \% \\
\hline Alto & 81 & 18 \\
Medio & 264 & 59 \\
Bajo & 103 & 23 \\
\hline \multicolumn{1}{c}{ Total } & 448 & 100 \\
\hline
\end{tabular}

\section{Diagnóstico y comparativa de resiliencia según género}

En el conglomerado, del $100 \%(n i=254)$ de estudiantes varones: de este sub grupo, el $20 \%$ (52) se autopercibe con resiliencia de nivel alto, el $56 \%$ (143) obtuvo puntuaciones que reflejan una resiliencia de nivel medio y, el $24 \%$ (59) obtuvo calificaciones que describen la pre- 
sencia de un nivel bajo de resiliencia, considerada como una fortaleza personal. Según la Tabla 4, el conglomerado hallado, del $100 \%($ ni = 194) de estudiantes mujeres: de este sub grupo, el $15 \%$ (29) se autopercibe con resiliencia de nivel alto, el $62 \%$ (121) obtuvo puntuaciones que reflejan una resiliencia de nivel medio y el $23 \%$ (44) obtuvo calificaciones que describen la presencia de un nivel bajo de resiliencia, considerada como una fortaleza personal.

Al procesar estadísticamente los datos de la resiliencia de varones y mujeres del grupo investigado, a través de la Prueba No paramétrica de la Ji Cuadrada de Pearson, se obtuvo una Jic $=2,56$ que comparado con la Jit $=5,99$ con gl. $=2$ y $\alpha=0,05$; permite establecer que no existen diferencias estadísticamente significativas en la resiliencia de los estudiantes investigación en función del género.

Tabla 4

Niveles de resiliencia en estudiantes de género masculino y femenino

\begin{tabular}{lcccc}
\hline \multirow{2}{*}{ Resiliencia } & \multicolumn{2}{c}{$\begin{array}{c}\text { Género masculino } \\
\text { global }\end{array}$} & $\begin{array}{c}\text { Género femenino } \\
\text { global }\end{array}$ \\
\cline { 2 - 5 } & Ni & $\%$ & Ni & $\%$ \\
\hline Alto & 52 & 20 & 29 & 15 \\
Medio & 143 & 56 & 121 & 62 \\
Bajo & 59 & 24 & 44 & 23 \\
\hline \multicolumn{1}{c}{ Total } & 254 & 100 & 194 & 100 \\
\hline
\end{tabular}

Diagnóstico y comparativa de resiliencia según tipo de gestión de la universidad

En el conglomerado global, del $100 \%(n i=140)$ de estudiantes encuestados de universidad privada: el $35 \%$ (49) se autopercibe con resiliencia de nivel alto, el 61 $\%$ (85) obtuvo puntuaciones que reflejan una resiliencia de nivel medio y, el $04 \%$ (06) obtuvo calificaciones que describen la presencia de un nivel bajo de resiliencia, considerada como una fortaleza personal. Según la Tabla 5, el conglomerado hallado, del $100 \%(n i=308)$ de estudiantes encuestados de universidad pública; el $10 \%$ (32) se autopercibe con resiliencia de nivel alto, el 58 $\%$ (179) obtuvo puntuaciones que reflejan una resiliencia de nivel medio y, el 32 \% (97) obtuvo calificaciones que describen la presencia de un nivel bajo de resiliencia considerada como una fortaleza personal.

$\mathrm{Al}$ procesar estadísticamente los datos de la resiliencia de estudiantes procedentes de universidad privada y estudiantes procedentes de universidad pública del grupo investigado, a través de la Prueba No paramétrica de la Ji Cuadrada de Pearson, se obtuvo una Jic $=63,34$ que comparado con la Jit $=5,99$ con gl. $=2$ y $\alpha=0,05$; permite establecer que si existen diferencias estadísticamente significativas en la resiliencia de los estudiantes investigación en función del tipo de gestión de la universidad de procedencia.
Tabla 5

Niveles de resiliencia en estudiantes de género masculino y femenino

\begin{tabular}{lcccc}
\hline \multirow{2}{*}{ Resiliencia } & \multicolumn{2}{c}{$\begin{array}{c}\text { Universidad privada } \\
\text { global }\end{array}$} & $\begin{array}{c}\text { Universidad pública } \\
\text { global }\end{array}$ \\
\cline { 2 - 5 } & $\mathbf{N i}$ & $\%$ & Ni & $\%$ \\
\hline Alto & 49 & 35 & 32 & 10 \\
Medio & 85 & 61 & 179 & 58 \\
Bajo & 06 & 4 & 97 & 32 \\
\hline \multicolumn{1}{c}{ Total } & 140 & 100 & 308 & 100 \\
\hline
\end{tabular}

\section{Discusión}

El estudio que se presenta, aborda el tema de la resiliencia humana considerando al enfoque ecológico de desarrollo humano. Este enfoque centra su esfuerzo en analizar el comportamiento atendiendo a su multicausalidad, en la que factores externos e internos se interconjugan, haciendo del ser humano un sujeto, cuyas cualidades y capacidades internas se ven fuertemente influenciadas por el entorno o contexto. En ese sentido, se busca entender al estudiante universitario como un ser dotado de fortalezas que le permitirían afrontar con éxito diversas dificultades o traumas que por cosas del destino le toca vivir. Por ende, en el marco de la ostentosa literatura previa revisada, se destaca ponderablemente el rol de los factores no cognitivos como las fortalezas del carácter y la resiliencia sobre el rendimiento académico y la implicación académica de los estudiantes.

En el contexto del estudio, se halló que la mayoría de los estudiantes universitarios de educación de la ciudad de Huancayo presentan nivel medio de resiliencia como fortaleza humana. Del mismo modo, se logró establecer que el tipo de gestión de la universidad (privada o pública) si juega un papel influyente sobre el perfil de resiliencia de los estudiantes investigados. La prueba estadística de la Ji Cuadrada permitió establecer un mejor perfil de resiliencia en los estudiantes procedentes de universidad privada.

Estos resultados logrados en el colectivo estudiantil investigado, estaría mostrando algunas debilidades o carencias de estos para enfrentar con éxito los acontecimientos adversos que les toca vivir, sobre todo en los estudiantes que cursan estudios profesionales en la universidad de gestión pública.

Los resultados encontrados en los estudiantes de la facultad de educación de la ciudad de Huancayo se contraponen a los hallados por otros investigadores, como Caldera, Aceves y Reynoso (2016), quienes abordaron resiliencia en estudiantes universitarios de Jalisco, México: Un estudio comparado entre carreras y logran concluir que la resiliencia alta es una característica muy generalizada en la mayoría de los estudiantes universitarios. 
El problema de los niveles bajos de rendimiento escolar y el abandono en el contexto de la educación universitaria, representan, actualmente, una de las problemáticas socioeducativas de mayor relevancia a la hora de plantear políticas de estrategias de mejora educativa. Para Díaz y Osuna (2017), abatir esta compleja problemática exige profundizar en los contextos y factores que la afectan y determinan. Por ello, se considera relevante profundizar en aspectos críticos del comportamiento humano que dan respuesta a los distintos escenarios vitales, principalmente en aquellas circunstancias que, por múltiples motivos, desbordan y parecen insalvables.

Se hace impostergable intervenciones en la resiliencia desde la educación y los agentes socializadores, de tal forma que el sistema educativo trabaje con propósitos mancomunados con la familia y otros agentes educativos, con el fin de mejorar el bienestar de sus estudiantes.

El tema de la resiliencia, como factor de comportamiento humano, está actualmente concitando el interés de la comunidad científica, pues diversos estudios, realizados en diversos escenarios, dan cuenta de este factor como un componente psicológico que ayuda a las personas a superar situaciones adversas.

Desde la prisma de la resiliencia, puede observarse que son muchas las personas que, pese a haber sufrido acontecimientos difíciles o traumas, estas cuentan con la gran capacidad para revertir exitosamente estas situaciones adversas. Según la literatura previa, la resiliencia debe ser concebida ante todo como resultado de un equilibrio entre factores de riesgo, factores protectores y personalidad de cada individuo. Otro aspecto que se rescata de esta literatura, es que se debe asumir que la psicología no debería centrarse solo en el estudio de la enfermedad (enfoque clínico o patológico), la debilidad y el daño, sino que también debe ahondar y privilegiar en el estudio de la fortaleza y la virtud del ser humano.

Al parecer, la resiliencia, en estos casos, se estaría mostrando más como factor que estaría amortiguando situaciones de ansiedad y estrés en los seres humanos permitiendo una buena adaptación y ajuste en las personas. lo planteado permite entender que las personas altamente resilientes son aquellas que se adaptan con facilidad a entornos deprimentes y acuciados por situaciones adversas.

Para Morelato (2014) "La resiliencia es la capacidad del ser humano para hacer frente a las adversidades de la vida, superarlas e inclusive ser transformado positivamente por ellas $(. .$.$) hace referencia al logro de una$ adaptación positiva o el afrontamiento adecuado de las tareas de desarrollo típicas de una determinada etapa o cultura, a pesar de las experiencias de adversidad” (p.56).
La resiliencia, como característica de la personalidad, hace que las personas tengan una mirada positiva y propositiva de las cosas y los acontecimientos, lo que permite que las personas mantengan y sostengan adecuadamente un proyecto de vida a nivel personal, profesional, familiar, etc.

Pues a partir de los esbozos teóricos de la resiliencia cabe la posibilidad que las personas, en este caso los estudiantes, tengan una vida mucha más saludable en un entorno insano, lleno de desvaríos e infortunios. La capacidad resiliente ofrece el potencial para superar adecuadamente los efectos negativos producidos por la exposición al riesgo mediante estrategias positivas de fortalecimiento y afrontamiento.

\section{Conclusiones}

- La mayoría de los estudiantes universitarios de educación de la ciudad de Huancayo presentan nivel medio de resiliencia como fortaleza humana. Se logra establecer que el tipo de gestión de la universidad (privada o pública) si juega un papel influyente y diferenciador sobre el perfil de resiliencia de los estudiantes investigados.

- La prueba estadística de la Ji Cuadrada permitió establecer un mejor perfil de resiliencia en los estudiantes procedentes de universidad privada. El género no se muestra como un factor diferenciador en la resiliencia de los estudiantes universitarios de educación investigados. Tanto varones como mujeres, del grupo investigado, presentan mayoritariamente niveles medios de resiliencia como cualidad personal.

\section{Referencias bibliográficas}

Börkan, B., Erkman, F. \& Serbest, S. (2016). Resilience as a mediator between parental acceptance - Rejection and depressive symptoms among university students in Turkey. Journal of Counseling and De-velopment, 94(2), 195-209.

Cabrera, V., Aya, V. y Guevara, I. (2014). Diseño y análisis psicométrico de una escala de resiliencia. Revista Facultad de Medicina, 62(2), 213-220.

Gómez, B. (2010). Resiliencia individual y familiar. Disponible en: http://www.avntfevntf.com/ imagenes/ biblioteca/G\%C3\%B3mez,\%20B.\%20Trab.\%20 3\%C2\%BA\%20BI\%2009-10.pdf

Grotberg, E. (1995). A guide to promotig resilience in children: strenghening the human spirit. The Internacional Resilence Proyect. La Haya, Holanda.

Gutiérrez, M. y Romero, I. (2014). Resiliencia, bien estar subjetivo y actitudes de los adolescentes hacia el consumo de drogas en Angola. Anales de Psicología, 30(2), 608-619. doi:http://dx.doi. org/10.6018/analesps.30.2.148131 
Heilemann, M.V., Lee, K. y Kury, F.S. (2003). Psychometric properties of the spanish version of the Resilience Scale. Journal of Nursing Measurement, 11(1), 61-72.

Hernández, R.; Fernández, C. y Baptista, M. (2010). Metodología de la investigación. Edit. McGraw- Hill. México.

Portella, A. y Liberales, A. (2015). Resilience in aging: literature review. Revista, Ciencia y Saúde Coletiva, 20(5), 1475-1495.

Psicogente, 19 (36): pp. 227, (2016). Revista Universidad Simón Bolivar. Barranquilla, Colombia. ISSN 0124 0137 EISSN 2027-212X.

Pimentel, C. y Pinho, L. (2015). Resiliencia en estudiantes universitarios. Un estudio comparado entre carreras.
Tesis de pregrado.

Quezada, L.; González, T. y Mecott, G. (2014). Resiliencia en pacientes pediátricos sobrevivientes de quemaduras. Acta de Investigación Psicológica, 4(2), $1585-1594$.

Vera, D. y López, M. (2014). Resiliencia académica: Una alternativa a explorar en la educación de niños y niñas en contextos rurales. Integra Educativa, 7(2), 187-205.

Wagnild, G. M. y Young, H. M. (1993) Development and psychometric evaluation of the Resilience Scale. Edit. Journal of Nursing Measurement, EEUU.

Wang, Y.; Chen, X.; Gong, J. y Yan, Y. (2016). Relation ships between stress, negative emotions, resilience, and smoking: Testing a moderated mediation model. Edit. Tesis Misuse. 